\title{
MESH MODELLING OF 3D POINT CLOUD FROM UAV IMAGES BY POINT CLASSIFICATION AND GEOMETRIC CONSTRAINTS
}

\author{
S. $\operatorname{Kim}^{1}$, H. G. Kim ${ }^{1}$, T. Kim,* \\ 1 3DLabs Co. Ltd., 100 Inharo, Namgu, Incheon, Korea - (shkim, khanai)@3dlabs.co.kr \\ ${ }^{2}$ Dept. of Geoinformatic Engineering, Inha University, 100 Inharo, Namgu, Incheon, Korea - tezid@inha.ac.kr
}

Commission II, WG II/3

KEY WORDS: Surface Reconstruction, Surface Normal, Point cloud, Mesh model

\begin{abstract}
:
The point cloud generated by multiple image matching is classified as an unstructured point cloud because it is not regularly point spaced and has multiple viewpoints. The surface reconstruction technique is used to generate mesh model using unstructured point clouds. In the surface reconstruction process, it is important to calculate correct surface normals. The point cloud extracted from multi images contains position and color information of point as well as geometric information of images used in the step of point cloud generation. Thus, the surface normal estimation based on the geometric constraints is possible. However, there is a possibility that a direction of the surface normal is incorrectly estimated by noisy vertical area of the point cloud. In this paper, we propose an improved method to estimate surface normals of the vertical points within an unstructured point cloud. The proposed method detects the vertical points, adjust their normal vectors by analyzing surface normals of nearest neighbors. As a result, we have found almost all vertical points through point type classification, detected the points with wrong normal vectors and corrected the direction of the normal vectors. We compared the quality of mesh models generated with corrected surface normals and uncorrected surface normals. Result of comparison showed that our method could correct wrong surface normal successfully of vertical points and improve the quality of the mesh model.
\end{abstract}

\section{INTRODUCTION}

As the performance of UAV (Unmanned Aerial vehicle) sensors and image processing technology are improved, UAVs are utilized more widely in remote sensing and aerial surveying. A UAV mapping system is economic and easy to operate compared with aerial photogrammetry system. Research on UAV photogrammetry for spatial information generation is actively proceeding. In order to generate spatial information using multiple images captured by UAVs, the following procedure should be performed: camera geometry estimation, $3 \mathrm{D}$ point cloud extraction, and 3D model generation.

From camera geometry estimation, many UAV image-based applications use the method called incremental bundle adjustment to estimate orientation of multi images. This method uses corresponding points created by feature matching from multi image pairs. For 3D point cloud extraction, there are many methods, such as PMVS, SURE, and so on. PMVS is a matching algorithm based on region growing that uses patchbased MVS algorithm. SURE is based on semi-global matching (SGM). These methods can generate precise point cloud and filter out noise point using geometric constraints of multiple stereo matching. The point cloud generated by multiple image matching is called unstructured point cloud because it is not on regular point spacing and has multiple viewpoints. Here, viewpoint means the position of camera sensor in coordinates of point cloud (Kim, 2017).

For 3D model generation, poisson surface reconstruction technique is mainly used to create meshes with unstructured point clouds. Since this technique generates mesh models by estimating surface using surface normals of input points, it is necessary to get accurate surface normals. It has advantages of being flexible to noisy data and constructing surface in a large hole with an empty point. A general pipeline of surface reconstruction consists of point cloud process, surface normal estimation, and mesh model generation. The surface normal estimation calculates normal value using neighborhoods of each point and adjusts normal direction using the viewpoint.

If surface normal vectors are calculated incorrectly and do not satisfy geometric constraints, a surface is adjusted in the wrong direction. Figure 1 shows surface normal vectors adjusted to wrong direction. In the noisy point cloud of figure 1, calculated surface normal of vertical point (red) is not correct even when it satisfied the geometric constraints. Therefore, the direction of normal is not adjusted. If the points with the wrong normal vectors are densely distributed, the quality of the generated mesh model is not good.

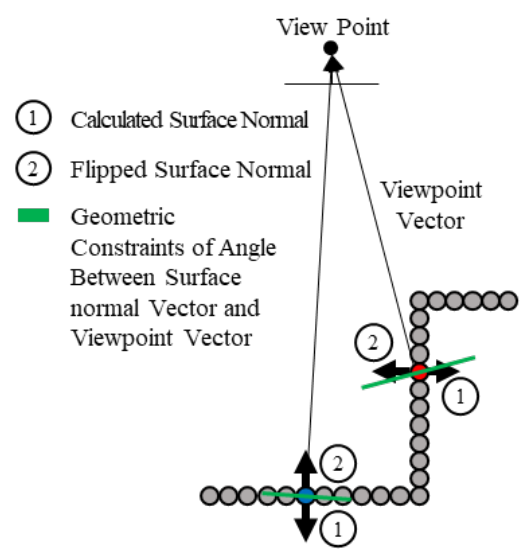

(a) True point cloud

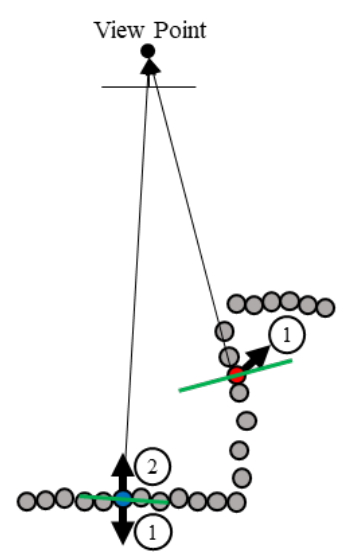

(b) Noisy point cloud
Figure 1. Adjustment of surface normal direction using geometric constraints

Vertical points (points located on a vertical plane) in a point cloud have lower density and are more noise than horizontal points (points located on a horizontal plane). Therefore, there is a possibility that surface normal vector is not calculated 
accurately. In addition, geometric constraints using the angle between viewpoint vector and surface normal vector of point is limited for vertical points. Thus, the direction of surface normal vector is often misadjusted at the vertical point. In order to solve this problem, it is necessary to classify the vertical points and analyze direction of surface normal vector to adjust to the right direction.

Jordan and Mordohai (2014) extended a study of Klasing et al. (2009) by considering three modifications to estimate accurate surface normal. They reported that it is more effective to use statistics of neighboring points than using the method to spread the surface normal of the reference point

Holzer et al. (2012) explained that the size of the set of neighbors has a significant effect on the accuracy of the surface normal. Therefore, in order to set adaptive size, they used outline extracted from the color image and depth map.
Previous studies have focused on the point cloud of 3D model objects extracted from active sensors. They used the statistics information of points. Thus, it is required to estimate accurate surface normals of point cloud extracted from multi images.

In this study, we propose a method to estimate the surface normal vectors of a point cloud based on geometric constraints and to correct estimated normal vectors of vertical points. In addition, we generate mesh models using the corrected surface normal vector and will confirm whether the quality is improved.

This paper is structured as follows. Section 2 explains theories related with process of typical mesh modelling. Section 3 introduces the proposed method to adjust wrong surface normal of vertical points. The research result is described in Section 4. Section 5 discusses conclusions and directions for future study.

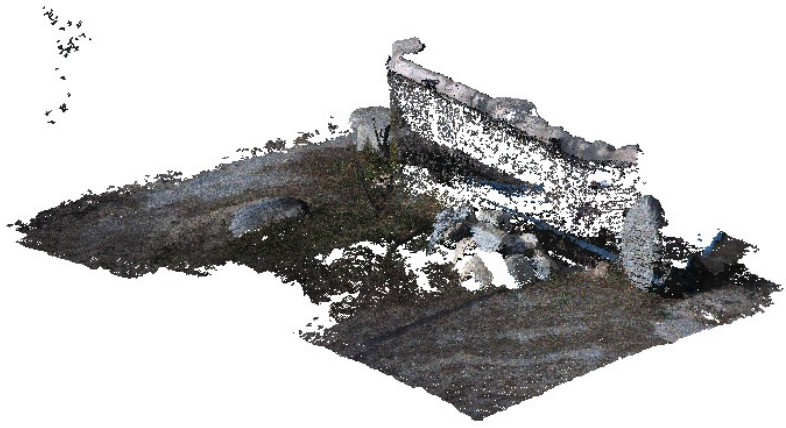

(a)

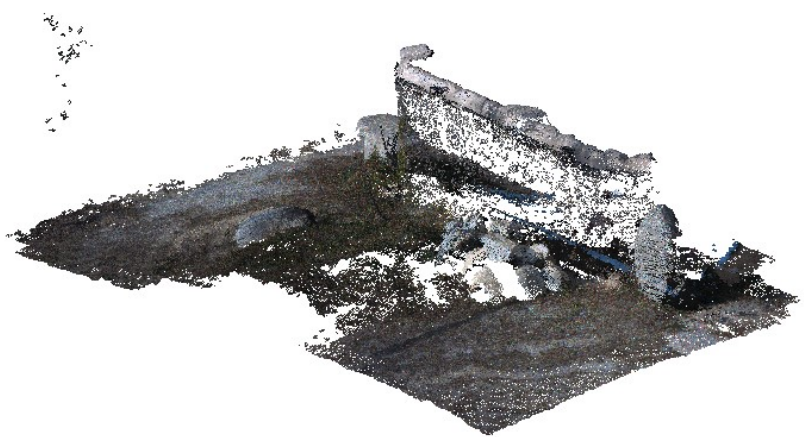

(b)

Figure 2. (a) Integrated point cloud, (b) Integrated point cloud with voxel grid filtering

\section{THEORIES}

\subsection{Point Cloud Filtering}

There are two types of point clouds; structured and unstructured type. Point clouds obtained from passive sensors such as multi images are unstructured ones. These point cloud typically has varying point densities and includes sparse outlier points due to image matching error. This complicates the estimation of local point cloud characteristics such as surface normal. This also decreases the quality of mesh models generated with point cloud (Rusu, 2008).

The method to generate point cloud extraction from multiple images is usually used multi image matching technique or integrating multiple point clouds extracted from stereo image matching. In the case of integrated point cloud, there are duplicate points for the area of overlap multiple images.

The voxel grid filtering creates a 3D voxel grid over the input point cloud data. Then, in each voxel, the point nearest to the center of the points in the voxel is extracted and the remaining points are removed as the duplicated points. The size of the voxel can be calculated from the average point spacing of the point cloud data before integrating to maintain the point density of the original point cloud. Figure 2 shows the results of applying voxel grid filtering to the integrated point cloud. (a) is the integration of 10 point cloud data and (b) is the result of the removal of the duplicated points in integrated point cloud. The average point spacing of the original point cloud was $0.020 \mathrm{~m}$ and integrated point cloud was $0.022 \mathrm{~m}$. In the Figure 2, the number of voxel grid filtered point clouds has decreased from
763,704 to 671,337 points. However, the density was maintained and the quality was not significantly different.

\subsection{Surface Normal Estimation based on Geometric Constraints}

Surface normal estimation process consists of surface normal calculation and surface normal adjusting. In general, surface normal is calculated after mesh generation. However, it is also used to generate the mesh model by estimating the surface normal using the surrounding points in the point cloud before the mesh generation. This method computes covariance matrix from the nearest neighbors and performs PCA (Principal Component Analysis). The direction of the surface normal extracted through PCA may be inconsistent because it is determined using only the surrounding points. For this reason, incorrect direction of the surface normal is adjusted using the geometric constraints so that it is consistently collocated towards viewpoint (Kim, 2017)

\section{PROPOSED METHOD}

In this paper, we propose a mesh modelling process in 5 steps: 1) Point cloud filtering and integration, 2) Calculating surface normal vectors of point cloud, 3) Adjusting wrong direction of the surface normal vectors, 4) Classifying vertical points, and 5) Correcting surface normal vectors of vertical points. Steps 1, 2, and 3 are typical mesh model generation process. Steps 3 and 4 are newly proposed to estimate correct surface normal vectors. 
Figure 3 shows the process of the mesh modelling using the proposed method.

In the first step, Input point cloud from each pair is merged into one total point cloud. Then point cloud filtering is performed to remove outlier and duplicate points.

In the fourth step, vertical points must be extracted from integrated point cloud. The integrated point cloud is classified into vertical points, horizontal points, and un-classified points by analyzing angles between surface normal vector of point and $\mathrm{Z}$-axis vector. If there are many vertical points around an unclassified point, it is classified as a vertical point. .

In the fifth step, wrong normal vectors of the vertical point are adjusted to the right direction. The surface normal vector of vertical point is re-assigned as that of the nearest vertical point. In order to do it, first, angles between the normal vector of the vertical point and normal vectors of surrounding points are calculated. If there are many angles with nearly opposite direction, it is determined that the direction of the normal vector of the vertical point is wrong. Then the normal vector is adjusted in the opposite direction.

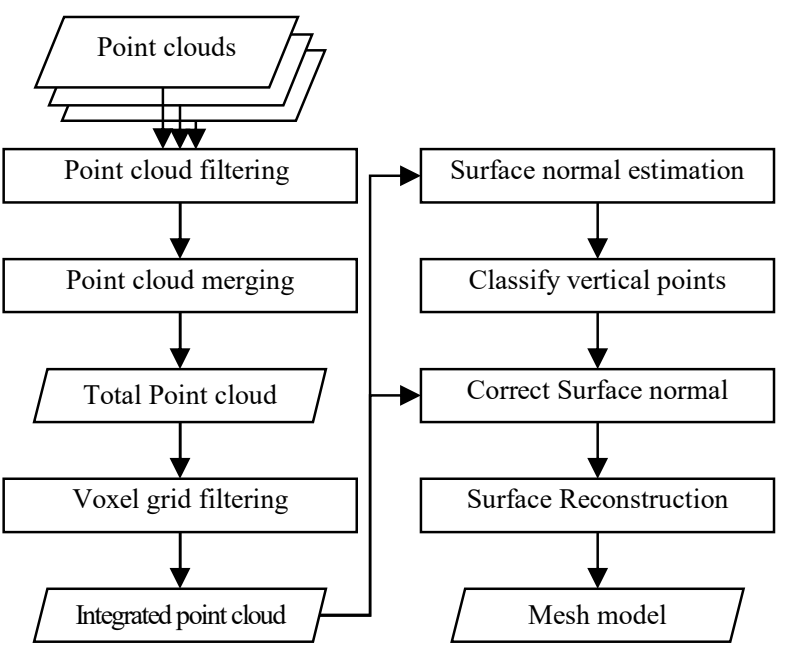

Figure 3. Mesh modelling process using proposed method

\section{EXPERIMENTS}

\subsection{Dataset}

The point cloud data for experiments were extracted from 10 stereo pair images. These images were acquired with a UAV equipped with a stereo camera. The UAV system consisted of stereo camera, RTK(Real Time Kinematic) GPS, and INS(Inertial Navigation System). It provided exterior orientation information of UAV in real time when taking images. Depth maps were generated from stereo images using stereo image matching. Then, direct georeferencing was performed to convert the depth map to the point cloud by using the exterior orientation information. As a result, all point cloud data had the same coordinate system. Table 1 shows the specification of UAV system. The position accuracy of RTK GPS was less than $0.05 \mathrm{~m}$ and rotation accuracy was less than 0.18 degree.

Input point cloud data acquired from the UAV system contained position and color information of point as well as geometric information of images used in the point cloud generation. The geometric information was used to surface normal estimation and classify point cloud.

\begin{tabular}{|c|c|c|}
\hline \multicolumn{2}{|c|}{ Type } & Detailed Spec. \\
\hline \multirow{4}{*}{ UAV } & Model & Umacair-D12 \\
\hline \multirow{4}{*}{ Camera } & Model & GS3-U3-120S6C-C \\
\cline { 2 - 3 } & Focal Length(mm) & 16 \\
\cline { 2 - 3 } & CCD Size( $\mu \mathrm{m})$ & 3.1 \\
\cline { 2 - 3 } & Image Size(px) & $4240 \times 2948$ \\
\hline \multirow{4}{*}{ GPS } & Manufacturer & Trimble \\
\cline { 2 - 3 } & Position(m) & $0.02-0.05$ \\
\cline { 2 - 3 } & Roll \& Pitch(deg) & 2 \\
\cline { 2 - 3 } & True Heading(deg) & 5 \\
\hline
\end{tabular}

Table 1. Specification of UAV system

\subsection{Result of Classifying Vertical Points}

We classified the integrated point cloud by calculating the angle between surface normal of point and Z-axis vector. If the angle was within $20^{\circ}$, it was classified as a plane point. If it was over $70^{\circ}$, it was classified as a vertical point. The rest were set as un-classified. Next, if the ratio of the vertical points among the surrounding points of the un-classified point was $70 \%$ or more, it was classified as a vertical point and its surface normal vector was re-assigned as that of the nearest vertical point. The process of classifying unknown points was repeated three times. Table 2 shows the number of un-classified points and vertical points as a result of performing the repeated process. In this result, we confirmed that the 342 out of the un-classified points were classified to vertical points. 'Init' of field in Table 2 is the result of the initial classification with the point cloud.

\begin{tabular}{|c|c|c|c|c|}
\hline Repeated times & Init & 1 & 2 & 3 \\
\hline $\begin{array}{c}\text { Number of the } \\
\text { point cloud }\end{array}$ & \multicolumn{4}{|c|}{671,337} \\
\hline $\begin{array}{c}\text { Number of the } \\
\text { horizontal point }\end{array}$ & 483,120 & 483,120 & 483,120 & 483,120 \\
\hline $\begin{array}{c}\text { Number of the } \\
\text { vertical point }\end{array}$ & 32,523 & 32,811 & 32,856 & 32,865 \\
\hline $\begin{array}{c}\text { Number of the } \\
\text { un-classified point }\end{array}$ & 155,694 & 155,406 & 155,361 & 155,352 \\
\hline
\end{tabular}

Table 2. Number of points classified into three types

Figure 4 presents the result of classifying point cloud. (a) is the result of initial classification and (b) is the result of classification of unknown points. Point color means the types of classified points. Red is the vertical point, blue is the plane point, and green is the unknown point. As a result, we confirmed that the green points clustered on the vertical points area had been changed to red points. 


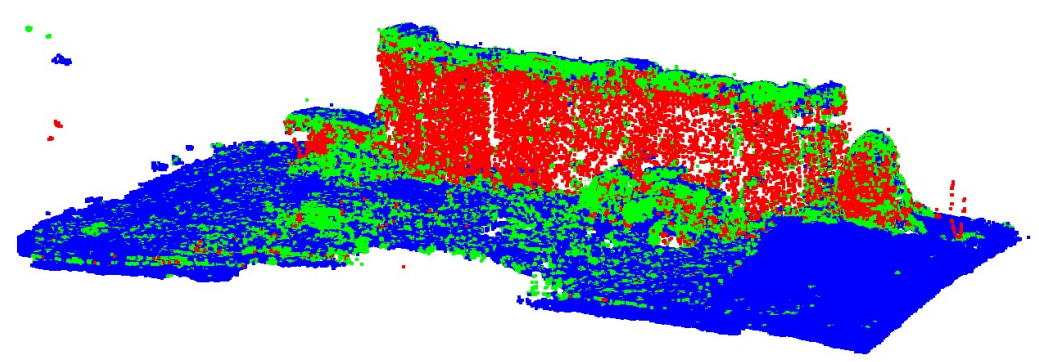

(a) Initial classification with the point cloud

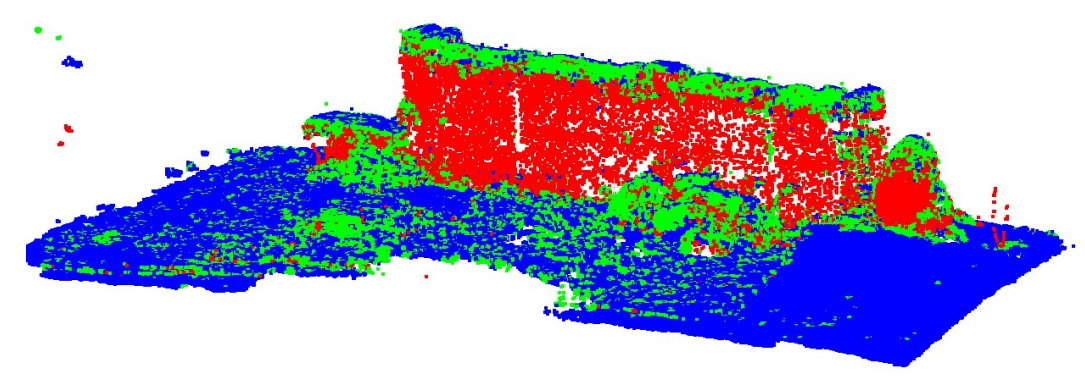

(b) Repeated classification with un-classified points

Figure 4. Result of classification with point cloud

\subsection{Result of mesh model with corrected surface normal}

In the surface reconstruction technique, if there are clustered points with wrong surface normals, distorted meshes are generated. In our experiments set, some points with wrong surface normal existed in the area of vertical points. We searched for vertical points with wrong surface normal, and we corrected the direction of the surface normal of the vertical points using our proposed method.

In order to search for the vertical points with wrong surface normal, we analyzed the ratio of the points with the angles between of the selected vertical point and neighboring vertical points higher than $50^{\circ}$. If the ratio of the analyzed points was lager than $50 \%$, we determined that selected point has a wrong surface normal. These points were adjusted to the opposite direction. The process was repeated three times to correct many wrong vertical points as possible.

Two mesh models were generated (Figure 5): one using the point cloud with uncorrected surface normal vector (Figure $5 \mathrm{a}$ ) and the other using the point cloud with corrected surface normal vectors (Figure 5b). After comparing the two mesh models, the mesh model with corrected normal vector showed better quality. In the Figure $5 \mathrm{a}$ and $\mathrm{b}$ images, meshes within the circles show the difference between the two models. We can check that the shape of the mesh model using corrected surface normal vector contained less artifacts.

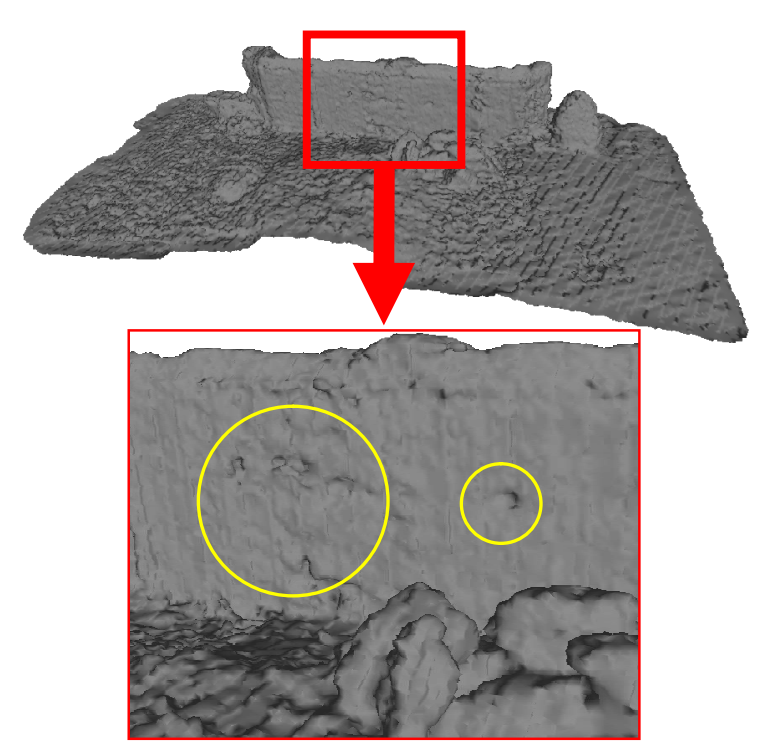

(a) Mesh model using uncorrected surface normal vector

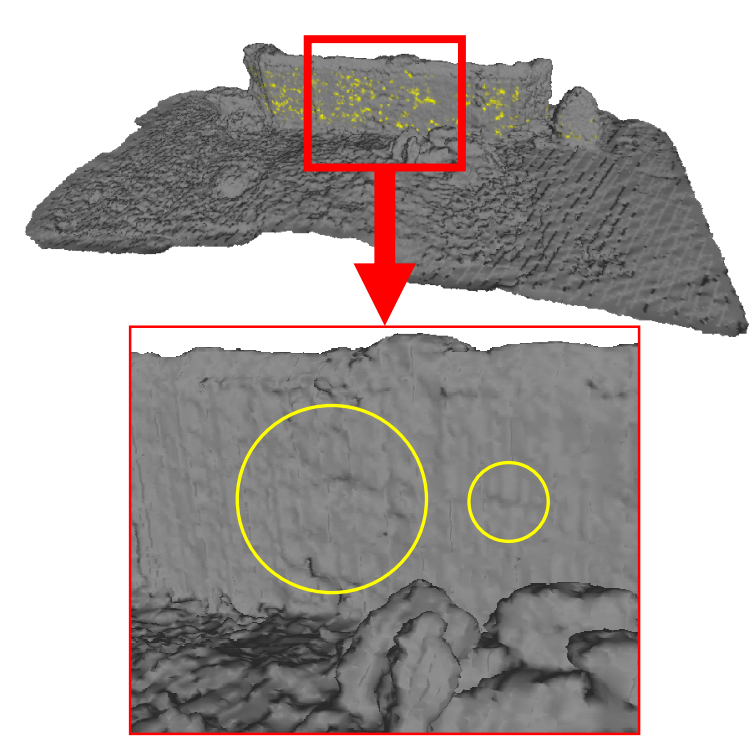

(b) Mesh model using corrected surface normal vector

Figure 5. Comparison of mesh models 


\section{CONCLUSION}

In this study, mesh models were generated using unstructured point cloud extracted from multiple UAV images. We improved the method of estimating surface normal vector by correcting the wrong surface normal vector of vertical points. As a result, we confirmed that the quality of the mesh model was improved by using the corrected surface normal vector. There were points uncorrected by the proposed method. Therefore, it is necessary to analyze these points and further study on the improvement.

\section{ACKNOWLEDGEMENTS}

This research was supported by a grant (18DRMS-B14728701) from Development of Realistic 3D Geospatial City Model Authoring and Providing Technology Program funded by Ministry of Land, Infrastructure and Transport of Korean government.

\section{REFERENCES}

Holzer, S., Rusu, R. B., Dixon, M., Gedikli, S., and Navab, N., 2012, Adaptive Neighborhood Selection for Real-time Surface Normal Estimation from Organized Point cloud data using Integral Images, IROS, pp. 2684-2689.

Jordan, K., Mordohai, P., 2014, A quantitative evaluation of surface normal estimation in point clouds, Intelligent Robots and Systems, 2014 IEEE/RSJ International Conference on.

Kazhdan M., 2006, Poisson Surface Reconstruction, Eurographics Symposium on Geometry Processing, pp. 61-70.

Kim, S., Kim, T., Improvement of Surface Reconstruction Pipeline using Open Source for Unstructed Point Cloud, 2017, International Symposium on Remote Sensing 2017, 62, pp. $119-121$.

Klasing, K., Althoff, D., Wollherr, D., and Buss, M., 2009, Comparison of surface normal estimation methods for range sensing applications, in IEEE International Conference on Robotics and Automation (ICRA). IEEE, pp. 3206-3211.

Rusu, R. B., Marton, Z. C., Blodow, N., Dolha, M., and Beetz, M., 2008, Towards 3D Point Cloud Based Object Maps for Household Environments, Robotics and Autonomous Systems Journal, 56(11), pp. 927-941. 